\title{
Determination of Sodium Benzoate in Dairy Products
}

\author{
Fang Liu ${ }^{*}, 1,2$, Qingqing Shen ${ }^{3}$, Honghui Chen ${ }^{1}$, Zhixin Zhang ${ }^{1}$, Xiaoqi Yang ${ }^{1}$ \\ ${ }^{1}$ College of Chemistry and Engineering, Wenshan University, Wenshan, Yunnan 663099, China; \\ ${ }^{2}$ Department of Chemistry, Tsinghua University, Beijing 100084, China; \\ ${ }^{3}$ College of Environment and Resources, Wenshan University, Wenshan, Yunnan 663099, China \\ *Corresponding Author’s Email: 2511284881@qq.com
}

Keywords: Dairy products; Benzoic acid; Determination

\begin{abstract}
To establish a simple and feasible pretreatment method for the determination of benzoic acid in dairy products by high performance liquid chromatography. Benzoic acid in dairy products was determined by high performance liquid chromatography after protein and fat were removed by precipitator. Benzoic acid showed a good linear relationship in the range of $0-30 \mathrm{mg} / \mathrm{L}(\mathrm{r}=0.9999)$. The detection limit was $0.2 \mathrm{mg} / \mathrm{L}$ benzoic acid. The key of this kind of sample treatment is how to remove protein completely without affecting the measurement results, that is, to find a more ideal precipitator. The dialysis method is used for the overnight dialysis treatment of the sample and then is determined by high performance liquid chromatography. The method is time consuming and is not conducive to the analysis and determination of a large number of samples. The recovery of the method is $91.1 \%$ to $101.2 \%$ of benzoic acid. The relative standard deviation of benzoic acid was $2.7 \%$. The concentration of sodium benzoate in the range of $0-2.5(\mathrm{~m} / / \mathrm{g})$ has a good linear relationship between $\mathrm{A}$ and concentration c. It can be quantitatively analyzed according to the standard curve method. The recovery rate of this method is $103.6 \%$, variation. The coefficient is less than 1.20 . The accuracy and precision of the method are satisfactory, and the operation is simple and easy. The method is simple, rapid, accurate and easy to operate, and is suitable for the determination of benzoic acid, sorbic acid and sodium saccharin in dairy products, and the results are satisfactory.
\end{abstract}

\section{Introduction}

In our country, dairy products gradually become the people's essential food. Since the reform and opening up, especially in recent years, China's dairy industry and dairy industry have developed rapidly. The production of dairy cattle, dairy products and dairy products has doubled. The consumption of dairy products has steadily increased. China has become the world's third largest milk producer after India and the United States.

Commercially available dairy products In order to maintain a long-lasting flavor and extend shelf life, manufacturers will add more or less food additives such as preservatives. Preservatives have the effect of inhibiting or killing microorganisms during the preservation of dairy products. It can make dairy products store at room temperature and under simple preservation conditions. So far, only 32 kinds of food preservatives have been approved in China, the most commonly used are benzoic acid, sorbic acid and so on. Benzoic acid is more toxic than sorbic acid, and its bacteriostatic effect is only $1 / 3$ of that of sorbic acid at the same acidity value, so many countries have gradually switched to sorbic acid. However, due to its low price, benzoic acid and its sodium salt are still used as main preservatives in China, mainly for liquid beverages. If the preservative content is within the scope of national standards, it will not harm health, but for long-term preservation of dairy products, manufacturers will blindly increase their dosage, because preservative is artificial, improper use will have a certain negative effect, long-term excessive consumption will cause certain damage to the health of consumers. Therefore, the detection of preservatives in food is particularly important [3]-[5].

At present, the detection methods of sodium benzoate in preservatives in China include: traditional chemical analysis method; gas chromatography, which has the characteristics of strong separation 
ability and high detection sensitivity; high performance liquid chromatography, which is simpler and does not require too much Extraction and purification; Ion exchange chromatography has the characteristics of simple pretreatment and fast analysis speed, but it has not been widely used and few reports have been reported. Infrared spectroscopy has the characteristics of nondestructive, rapid and convenient, which can provide a new analytical method for the determination of sodium benzoate in dairy products. In this experiment, the sodium benzoate in dairy products was determined by Fourier infrared absorption spectrometry.

\section{Instruments and samples}

\subsection{Instrument}

Fourier transform infrared spectrometer IRPrestige-21 (Shimadzu, Japan) wavenumber range 400-4000 cm-1, xs225A electronic balance, SSP-10A manual oil pump tablet press, agate bowl, mirror paper, disposable film gloves, infrared drying lamps, stainless steel sample spoons, capillary droppers, tweezers, drying ovens, glass rods, beakers, etc.

\subsection{Reagent}

Potassium bromide (spectral pure), distilled water, pure sodium benzoate analysis

Table 1 categories and sources of samples

\begin{tabular}{cc}
\hline Varieties of dairy products & Manufacturer \\
\hline Lysol pure milk & Yunnan huang's lysier dairy co. LTD \\
Lysol sour milk & \\
\hline Mengniu pure milk & Inner Mongolia Mengniu Dairy (Group) Co., Ltd. \\
Mengniu sour milk & \\
Mengniu Breakfast Milk & Yunnan Dali east Asia dairy co. LTD \\
\hline Eurasian strawberry milk & \\
beverage & \\
Eurasian pure milk & \\
Eurasian sour milk & \\
Eurasian breakfast milk & \\
\hline Sheran pure milk & Yunnan New Hope Dairy Holdings Co., Ltd. \\
Sheran peanut milk & \\
Sheran milk drink tastes of peach & \\
Sheran plain yogurt &
\end{tabular}

The above 15 dairy products were obtained at the Blair Mart shopping center in Wenshan.

\section{Sample processing and determination}

After being pressed, the pure potassium bromide of the spectrum was fixed on the scaffold, and then the sample -- dairy products were put into the sample quantitatively by capillary drip tube, and then measured by Fourier transform infrared spectrometer.

\section{Spectral processing}

The obtained sample spectra are processed by smoothing point 5, and then the characteristic absorption peaks are marked to obtain one-dimensional infrared spectra. Then the samples of different categories from the same manufacturer and the samples of different manufacturers from the same category are superimposed. 


\section{Results and discussion}

\subsection{Comparison of different types of dairy products in the same factory}

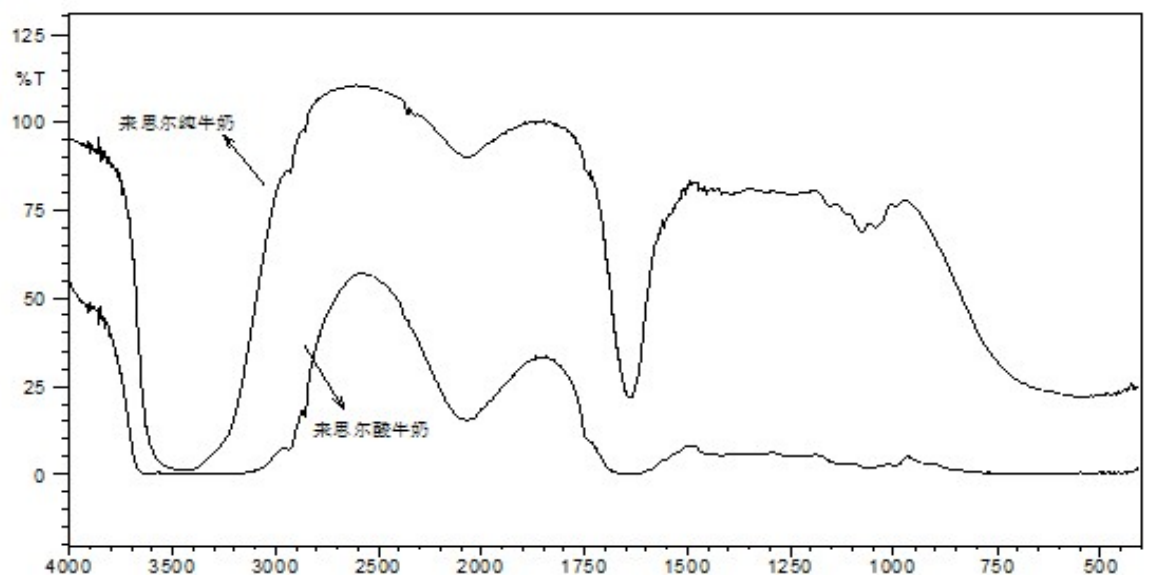

Figure 1. Overlapping dairy products of Lysol brand

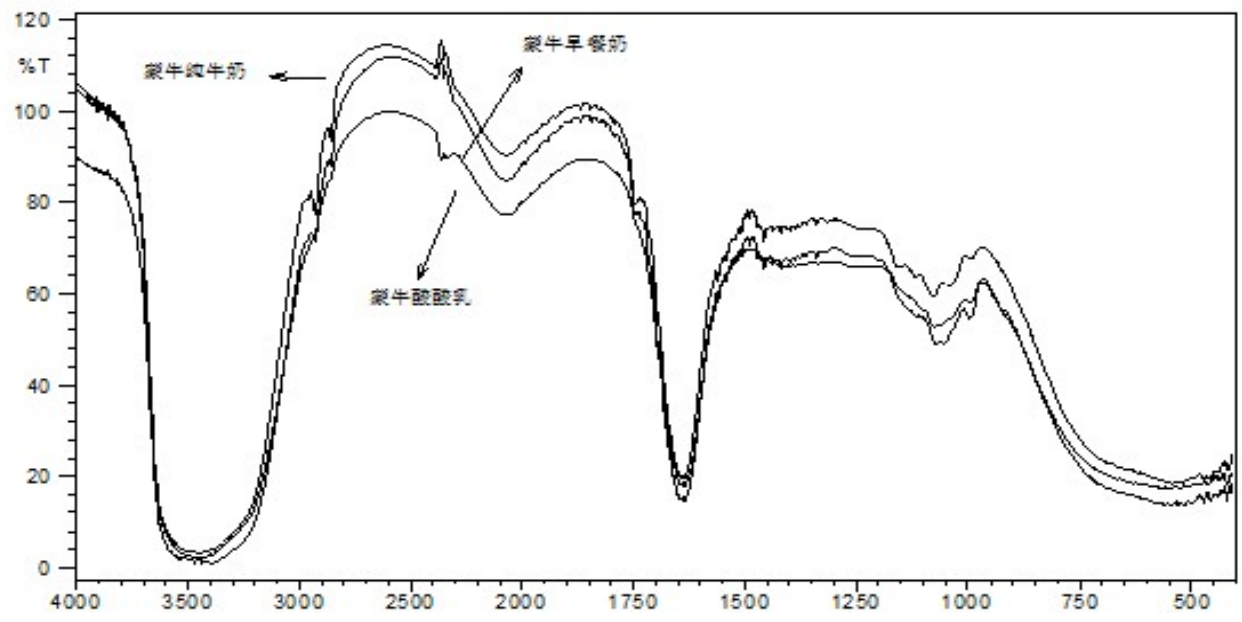

Figure 2. Overlapping images of Mengniu dairy products

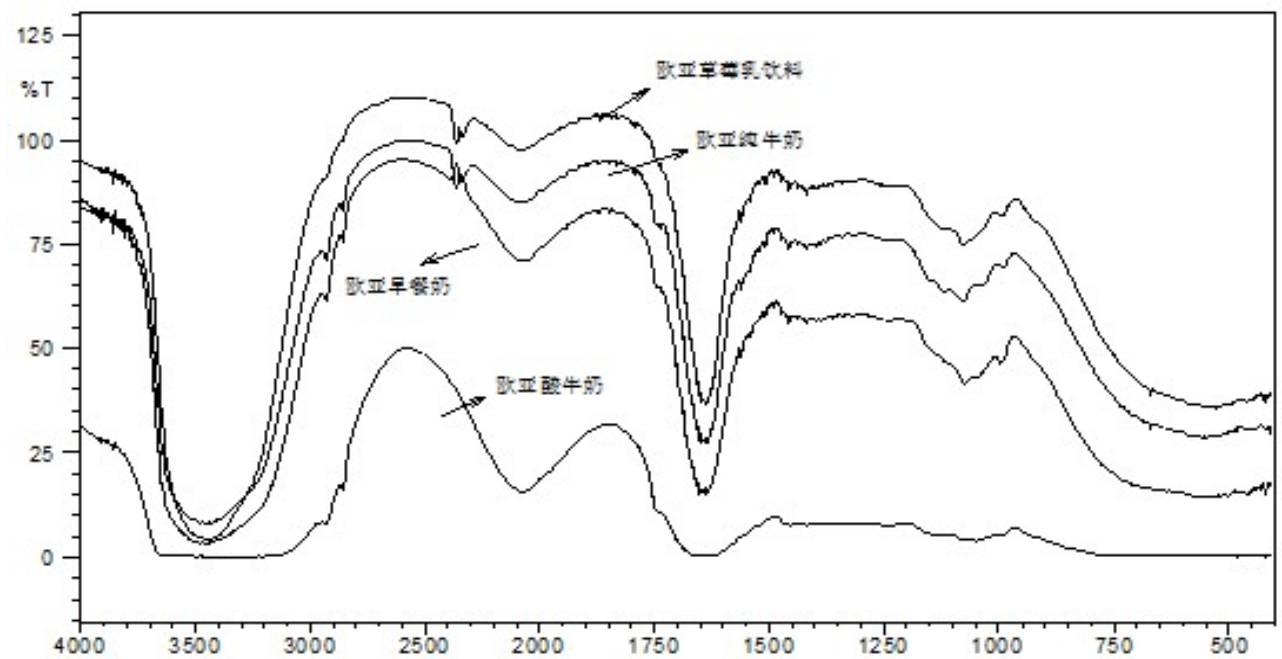

Figure 3. Dairy overlapping of Eurasian brands 


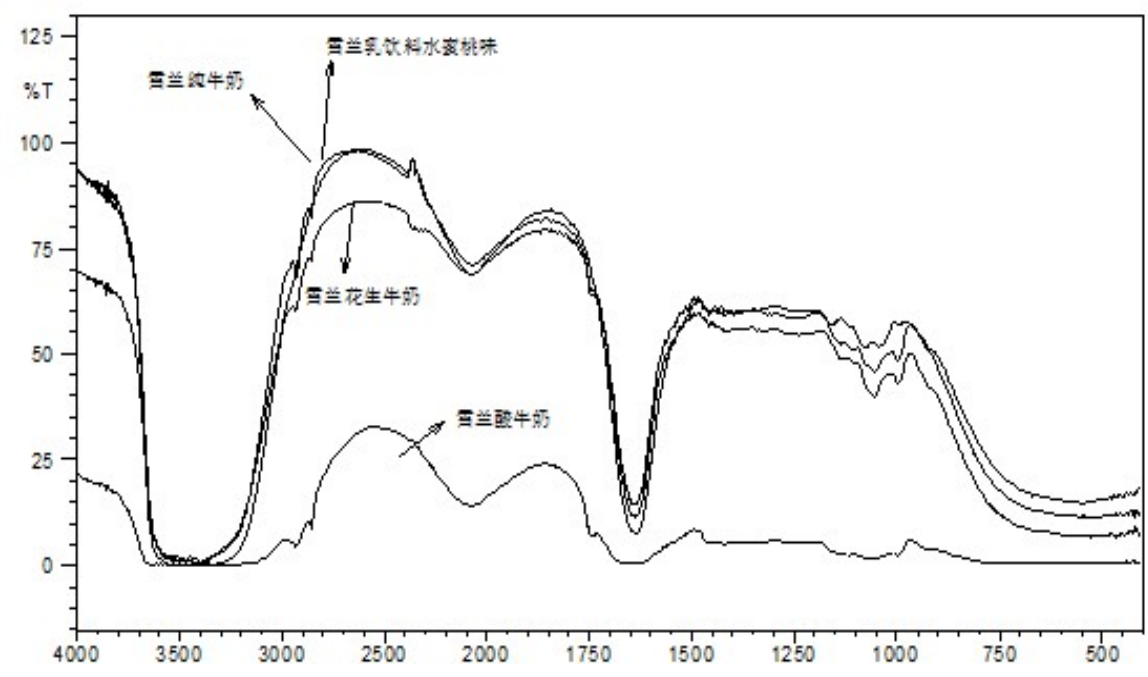

Figure 4. Overlapping images of Sheran dairy products

The infrared absorption spectrum of dairy products mainly shows the absorption peaks of water, protein and fat. Water is the most abundant substance in dairy products and the most absorbed compound. The absorption of water mainly comes from hydroxyl (- $\mathrm{OH})$. In the mid-infrared region, the absorption positions of water are 1543-1717 cm-1 and 2971-3627 cm-1, respectively. Protein molecule is a long chain molecule composed of amino acid molecule through peptide bond (-CO-NH-). The $\mathrm{N}-\mathrm{H}$ bond in the molecule is the main absorption group. The bending vibration absorption of $\mathrm{N}-\mathrm{H}$ and $\mathrm{C}-\mathrm{H}$ bonds in amide I band and amide II band at $1650 \mathrm{~cm}-1$ correspond to $\mathrm{C}=\mathrm{O}$ absorption peak and $1538 \mathrm{~cm}-1$ correspond to amide II band. The IR absorption of fatty molecules is mainly determined by $\mathrm{C}=\mathrm{O}$ bond in carboxyl group and saturated $\mathrm{C}-\mathrm{H}$ bond in fatty acid chain, including methylene (-CH), methylene (-CH2) and methyl (-CH3).The medium infrared fundamental frequency absorption position of the fat is respectively located at $1754 \mathrm{~cm}-1$, corresponding to the stretching vibration of the $\mathrm{C}=\mathrm{O}$ bond in the fat. This position is related to the number of fat molecules. $2857 \mathrm{~cm}-1$ corresponds to stretching vibration of saturated c-h bond in fatty acid chain. The bending vibration of the saturated c-h bond in the fatty acid chain corresponds to $1470 \mathrm{~cm}-1$.

Different brands of dairy products have corresponding absorption peaks in the three main components, but the absorption peaks of each brand of yoghurt are obviously different from other dairy products, because yoghurt is raw milk, after pretreatment and then access to pure culture of Lactobacillus bulgaricus and Streptococcus thermophilus as hair. A product that condenses casein for a certain period of time due to lactic acid production. During the fermentation process, about 20\% of the sugars and proteins in milk are broken down into small molecules (such as galactose and lactic acid, small peptide chains and amino acids). The fat content in fresh milk is generally $3 \%-5 \%$. After fermentation, the fatty acids in yoghurt increase twice as much as raw milk. These changes make yogurt more digestible and absorbable, and the utilization of various nutrients is improved. Yogurt is fermented by pure milk. In addition to retaining all the nutrients of fresh milk, lactic acid bacteria can also produce various vitamins necessary for human nutrition during fermentation, such as VB1, VB2, VB6, VB12 and so on. Therefore it exhibits a different absorption than other dairy products.

At the same time, according to the corresponding amides II belt of $1538 \mathrm{~cm}-1 \mathrm{~N}-\mathrm{H}$ and $\mathrm{C}-\mathrm{H}$ key bending vibration absorption strength as well as the corresponding to fat in the $\mathrm{C}=\mathrm{O}$ bond stretching vibration absorption strength of $1754 \mathrm{~cm}$ - 1 as you can see the Sheran brand of dairy products most pure milk protein and fat, milk beverage, the lowest is peanut milk; Eurasia brand dairy products have the highest protein and fat content of pure milk, followed by breakfast milk, the lowest is milk beverage; Mengniu brand dairy products have the highest protein and fat content of pure milk, followed by breakfast milk, the lowest is Sour milk.

By comparing the protein and fat content of the above dairy products, we can clearly see that although breakfast milk is now widely advertised and the products are coming out in a rush, the protein and fat content of breakfast milk is not as high as that of pure milk. This is due to their 
different ingredients. Pure milk uses fresh and high-quality milk, which is effectively heated and sterilized and sold in separate packages. Pure milk does not contain any excipients during processing. It is $100 \%$ milk. Breakfast milk is strictly treated with seasoned milk drinks. In order to make the milk delicious, dairy enterprises add some white granulated sugar, fruit juice, peanut, cereal and malt essence into the milk. While these additives make flavored milk more palatable, they dilute the milk itself, reducing both protein and fat. Therefore, from the perspective of protein and fat content, the nutrition of breakfast milk is definitely not as good as pure milk. But because other ingredients such as wheat and peanuts also contain a certain amount of protein, the overall reduction is not much.

\subsection{Determination of sodium benzoate in dairy products}

The characteristic analysis peak of 1552cm-1, 1552cm-1 was isolated and sharp from the infrared spectrum of sodium benzoate, while there was no peak for dairy products. Therefore, $1552 \mathrm{~cm}-1$ was selected as the analysis peak of this method. The absorbance of different concentrations of sodium benzoate was measured at the wave number, and the absorbance value was plotted on the ordinate, and the corresponding concentration was plotted on the abscissa, and the working curve was drawn. The absorbance of the sample to be tested was substituted into the regression equation to calculate the sodium benzoate content [7].

Sodium benzoate of different quality was accurately weighed in turn and a certain amount of potassium bromide was fully mixed in the abrasive bowl. $100 \mathrm{mg}$ mixed sample was pressed and its absorbance was determined by FTIR. The data are shown in Table 2:

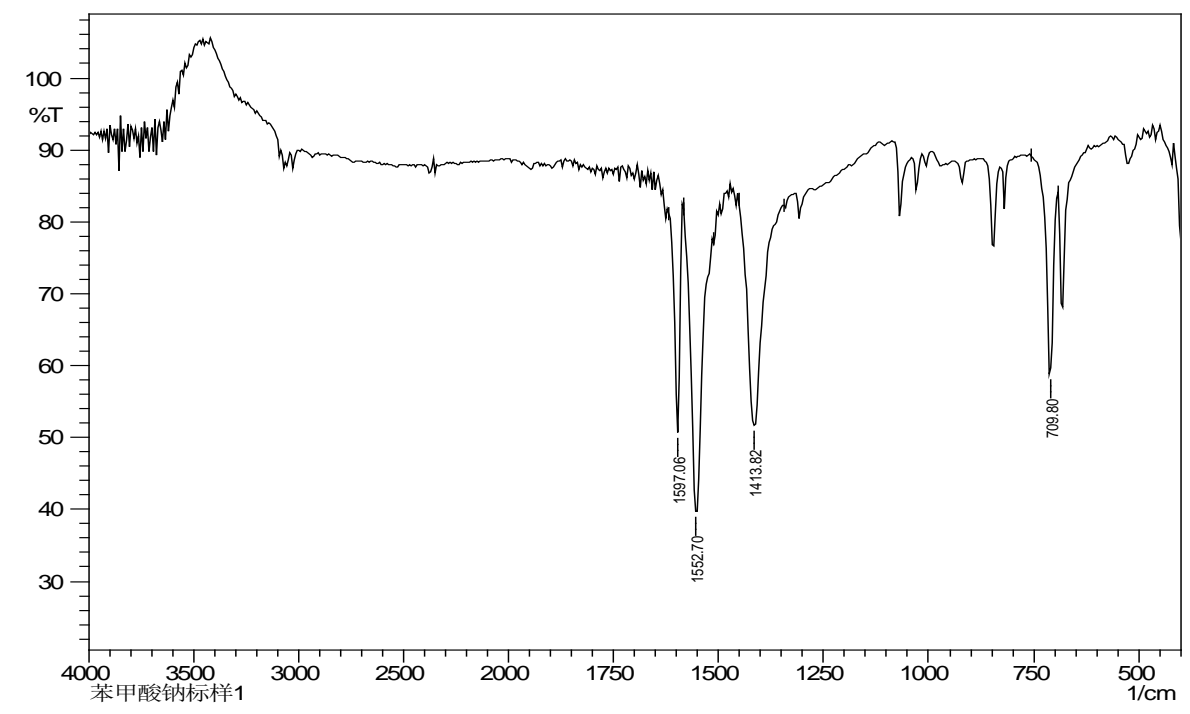

Figure 5 Sodium benzoate standard sample

Table 2 Working curve data

\begin{tabular}{ccccc}
\hline $\begin{array}{c}\text { Sodium } \\
\text { benzoate } \\
\text { standard } \\
\text { sample }\end{array}$ & $\begin{array}{c}\text { Quality of } \\
\text { Sodium } \\
\text { benzoate [mg] }\end{array}$ & $\begin{array}{c}\text { Quality of } \\
\text { potassium } \\
\text { bromide [mg] }\end{array}$ & $\begin{array}{c}\text { Sodium benzoate } \\
\text { content [mg/g] }\end{array}$ & $\begin{array}{c}\text { Absorbanc } \\
\text { e [A] }\end{array}$ \\
\hline Sample1 & 1.3 & 202.7 & 6.4 & 0.511 \\
Sample2 & 2.1 & 199.8 & 10.4 & 1.12 \\
Sample3 & 3.0 & 198.7 & 14.9 & 1.24 \\
Sample4 & 4.1 & 201.2 & 20.0 & 1.70 \\
Sample5 & 5.2 & 202.0 & 25.1 & 2.21 \\
Sample6 & 6.4 & 199.9 & 31.0 & 2.64 \\
\hline
\end{tabular}

The regression equation of the relation between $\mathrm{A}$ and concentration is as follows: $\mathrm{A}=0.0833 \mathrm{C}+$ 0.0731 A good linear standard curve can be drawn according to the relation between absorbance A and concentration C. The correlation coefficient $r=0.9915$ is obtained. The critical correlation 
coefficient $r(99 \%, n-2)=0.9170, r>r(99 \%, n-2)$ [7] is obtained by looking up the table, indicating that the concentration of sodium benzoate is $0 \sim$. There is a good linear relationship between A and concentration $\mathrm{C}$ in the range of 31.0 (mg/g), which can be quantitatively analyzed by the standard curve method.

\subsection{Determination of samples}

Table 3 Results of sample determination

\begin{tabular}{cccc}
\hline Sample & $\begin{array}{c}\text { Eurasian strawberry } \\
\text { milk beverage }\end{array}$ & $\begin{array}{c}\text { Eurasian } \\
\text { breakfast milk }\end{array}$ & $\begin{array}{c}\text { Eurasian pure } \\
\text { milk }\end{array}$ \\
\hline $\begin{array}{c}\text { AbsorbanceA } \\
\text { Sodium benzoate content } \\
{[\mathrm{mg} / \mathrm{g}]}\end{array}$ & 0.1052 & 0.1811 & 0.1569 \\
\hline
\end{tabular}

Table 4 experimental results of recovery rate

\begin{tabular}{cccc}
\hline $\begin{array}{c}\text { Sample } \\
\text { measurement(A) }\end{array}$ & Scaling amount(mg) & Scaling measurement(A) & $\begin{array}{c}\text { Recovery } \\
\text { rate(\%) }\end{array}$ \\
\hline 0.2681 & 0.005 & 0.273 & 98 \\
\hline
\end{tabular}

\section{Conclusion}

Based on the chemical structure and composition of dairy products, the absorption spectra of milk in the mid-infrared range were analyzed to extract the spectral characteristics of the main components of milk. The results showed that the difference of main components in different dairy products could be preliminarily distinguished by comparing the absorption position and intensity of the samples in the mid-infrared region. At the same time, the content of sodium benzoate in dairy products was determined. The method was simple and accurate, with a good linear relation of working curve, $\mathrm{r}=0.9915$, and the recovery rate was $98 \%$. According to the national standard, the sodium benzoate content in food is between $0 \mathrm{mg} / \mathrm{g}$ and $2 \mathrm{mg} / \mathrm{g}$, and the three samples tested in this test all meet the requirements of the national standard.

\section{References}

[1] Hongwei Zhang, Dongmei Zheng, Baohua Kong. Chemical and microbiological changes during storage of disinfectant on buses,[J]. China dairy industry, 2011, 49(10): 27-31.

[2] Junting Ji. Fruity milk beverage making process [Z].Shanhaiguan Food Factory, 19-20.

[3] Xu Hua. Development of breakfast milk [J].Chinese dairy industry, 2009, (1): 56-57.

[4] Jie Li. Strategic significance of dairy product quality and safety [J].Chinese dairy industry, 2012, (131): 20-23.

[5] Gaoqi Tang, Bin Cao. Food additive [M].China agricultural university press,2010: 12-13.

[6] Yulian Tang. Application of near infrared spectroscopy in rapid detection of components in dairy products [J].Dairy Science and technology, 2009,(4): 190-140.

[7] Ruihua Hui, Yan Dong, Xin Suo,etc. Determination of sodium benzoate in milk powder by FTIR [J].Food science,2003,24(8): 121-123. 